\title{
Multi-Trigger Resist for Electron Beam and Extreme Ultraviolet Lithography
}

\author{
C. Popescu, ${ }^{\mathrm{a}, \mathrm{b}}$, A. McClelland ${ }^{\mathrm{c}}$, D. Kazazis ${ }^{\mathrm{d}}$, G. Dawson ${ }^{\mathrm{c}}$, J. Roth ${ }^{\mathrm{e}}$, Y. Ekinci ${ }^{\mathrm{d}}$, W. Theis ${ }^{\mathrm{a}}$, A.P.G. \\ Robinson ${ }^{\mathrm{b}, \mathrm{c}}$ \\ ${ }^{a}$ Nanoscale Physics, Chemistry and Engineering Research Laboratory, University of Birmingham, \\ $U K$. \\ ${ }^{b}$ School of Chemical Engineering, University of Birmingham, UK. \\ ${ }^{c}$ Irresistible Materials Ltd, Birmingham, UK \\ ${ }^{d}$ Paul Scherrer Institute, 5232, Villigen, Switzerland \\ ${ }^{e}$ Nano-C, Westwood, MA, USA.
}

\begin{abstract}
The multi-trigger resist (MTR) is a new negative tone molecular resist platform for electron beam lithography, as well as extreme ultraviolet and optical lithography. The performance of xMT resist, the precursor to MTR resist, which shows a good combination of sensitivity, low line edge roughness and high-resolution patterning has previously been reported.[1] In order to overcome limitations induced by acid diffusion, a new mechanism - the multi-trigger concept — has been introduced. The results obtained so far as the behaviour of the resist is driven towards the multi-trigger regime by manipulating the resist formulation are presented. A feature size of $13 \mathrm{~nm}$ in semi-dense (1:1.5 line/space) patterns, and $22 \mathrm{~nm}$ diameter pillar patterns are demonstrated in electron beam, and $16 \mathrm{~nm}$ half-pitch resolution patterns are demonstrated in (extreme ultraviolet) EUV. An improvement in the LER value is seen in the higher MTR formulations.
\end{abstract}

Keywords: multi-trigger, molecular resist, quencher stochastics

\section{INTRODUCTION}

Remarkable progress has been made in the semiconductor industry with advances in integrated circuit technology. This has been driven by development of lithography techniques capable of high-resolution patterning, which include EUV, DUV, and electron beam, and also new generations of high-resolution high-sensitivity resist materials.

Extreme ultraviolet lithography (EUVL) at $13.5 \mathrm{~nm}$ is most likely to be the chosen post-optical technique for patterning sub-20-nm half-pitches for chip manufacturing, [2] with high-volume manufacturing (HVM) deployment expected imminently. Along with addressing issues regarding the availability of EUV power sources, the most challenging task to enable EUVL for high-volume production has been the need for improvement of resist performance or the development of novel resist materials capable of meeting industry requirements for ultimate resolution while maintaining a good sensitivity and low line edge roughness.[2]

Traditional resists have allowed a half-pitch of $22 \mathrm{~nm}$ in production to be achieved, and will likely support the initial HVM introduction of EUV. However, chemically amplified resists naturally limit the ultimate resolution in the resist due to acid diffusion and additionally post exposure instability in the patterned regions.[2] In addition, for sub-20-nm patterning the line edge roughness (LER)and line width roughness (LWR) become critical parameters that put an additional constraint on chemically amplified resists. Recently, attention has focused on the development of nonchemically amplified resists for future lithography nodes, such as an MAPDST-MMA copolymer that contains a sulfonium group to give the material sensitivity for electron beam radiation. A feature size of $20 \mathrm{~nm}$ in 1:2 line/space patterns was achieved.[2]

34th European Mask and Lithography Conference, edited by Uwe F.W. Behringer,

Jo Finders, Proc. of SPIE Vol. 10775, 1077502 - () 2018 SPIE

CCC code: $0277-786 X / 18 / \$ 18 \cdot$ doi: $10.1117 / 12.2316628$

Proc. of SPIE Vol. 10775 1077502-1 
In an MTR, multiple distinct chemical reactions must take place simultaneously and in close proximity for the chemical amplification process to proceed. Thus, at the edge of the feature, where the density of photo-initiators, such as photoacid generator (PAG) acids, that drive the chemical reactions is low, the amplification process is self-terminating, rather than requiring a quencher. This significantly reduces blurring effects and enables much improved resolution and LER while maintaining the sensitivity advantages of chemical amplification. MTR resist can provide a single resist solution to hybrid patterning approaches including i-line, $193 \mathrm{~nm}$, EUV and electron beam patterning.[3-5] This study presents results obtained where the strength of the multi-trigger effect is modulated through resist formulation changes.

\section{EXPERIMENTAL}

Resist samples were prepared by dissolving the individual components in ethyl lactate, and then purifying the samples using a metal ion reduction system. Resists were formulated with different weight ratios of these individual components to drive the resist behaviour in different directions. The baseline for the optimization is the previously introduced xMT resist system, shown in Fig. 1, from which the MTR1 series resist was developed. The resist was spun onto a commercial underlayer, Brewer Scientific E2 STACK AL412-302.

After the spin-coating of the resist, the samples received a post application bake (PAB) of $60{ }^{\circ} \mathrm{C}$ for 180 seconds. EUV exposures were performed using the XIL-II interference lithography tool [6,7] and electron beam patterning utilized a Vistec EBPG 5000+ electron beam exposure tool at $100 \mathrm{kV}$, both at the Paul Scherrer Institute, Switzerland. Exposures were also realized on ASML NXE3300 at IMEC using different custom illumination settings for line-and-space patterns (dipole-like). After exposure the samples did not receive a post exposure bake, and were developed in n-butyl acetate followed by a methyl isobutyl carbinol, (MIBC) rinse.

Exposed samples were analyzed with a scanning electron microscope (SEM) in top-down view. Critical dimension (CD) and LER were calculated from the SEM images with the commercial software package SuMMIT.

Samples exposed on the IMEC NXE 3300 in order to obtain unbiased roughness values, 50 square images of the pattern were taken at different position on one die and were then analyzed using metroLER ${ }^{\mathrm{TM}}$ software from Fractilia to remove SEM noise from the power spectral density (PSD) graphs (as per imec protocol cfr [8]).

As the direct measurement of the on-wafer dose for the EUV-IL exposures was not available in this study, the exposure was measured with respect to an internal PSI reference resist calibrated against exposures of the same resist at the Intel MET.[7] For features smaller than the capability of the calibration resist, the dose was estimated from higher pitch sizes.
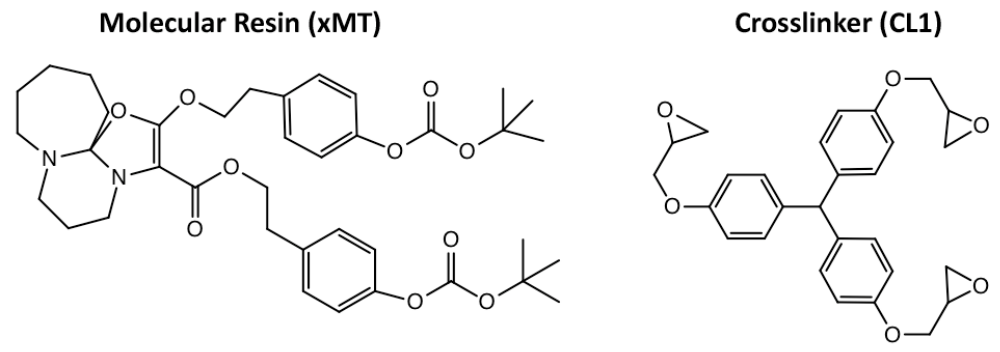

Photo-acid generator (PAG1)

Figure.2.1 Resist components in the baseline xMT system

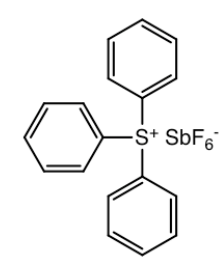

\section{RESULTS}

Formulations that demonstrate simultaneous high resolution, low LER and high sensitivity were investigated and the results are presented below. Alternative formulations were obtained by adjusting the components of the resist and their ratios. Through appropriate formulation it was possible to drive the behaviour of the resist system towards a response that was closer to a chemically amplified system, or towards the multi-trigger response. 


\subsection{Demonstrating MTR action}

To demonstrate the self-quenching behaviour described in the introduction the standard MTR formulation and the high MTR action formulation were exposed and analysed on the NXE 3300 exposure tool at IMEC.[9] Half pitches of 20 nm and $16 \mathrm{~nm}$ were successfully resolved and the LER value at $16 \mathrm{~nm}$ half pitch was monitored. It was observed that the sensitivity decreased with the MTR level but the unbiased value for LER improved (from $4.9 \mathrm{~nm}$ to $3.7 \mathrm{~nm}$ ), which shows that the material expresses a dose-dependent self-quenching behaviour that limits the acid diffusion length in low dose areas.

\subsection{MTR in electron beam lithography}

Although the MTR system was designed as part of the novel EUV resists platform its performance was also tested in electron beam lithography to get a good insight of what the mechanism of interactions during exposure might be and compare it to the observations acquired in EUV.

The advantage of using electron beam lithography is the fact that it is a mask-less technique, which means that the lithographic performance obtained is due entirely to the properties of the material and not the mask. The other reason electron beam is an advantageous technique in this study is that the number of electrons in the incident beam is larger than the number of photons in the EUV exposures. This becomes important when investigating the roughness due to stochastic events in the material such as quencher stochastics.

\subsection{The effect of additional quencher on the MTR system}

In order to understand what effect an additional quencher can have on the MTR system, the MTR level was varied and an external quencher was added in two different concentrations. The samples obtained were exposed to $100 \mathrm{kV}$ electron beam and patterns featuring lines of 14 and $18 \mathrm{~nm}$ at relaxed pitches were analysed. The results are summarised in Figure 3.2 where the normalised minimum LER is plotted as a function of the MTR ratio and the additional quencher concentration.

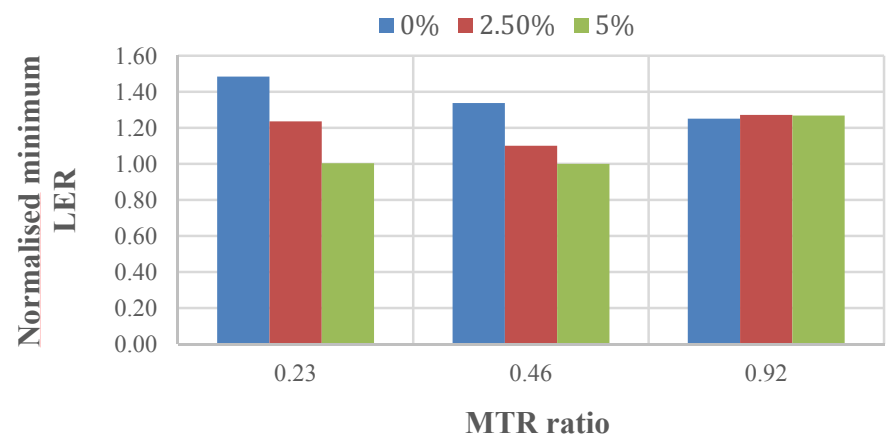

Figure 3.2. Effect on LER of changing MTR ratio and quencher level

It can be seen in Fig. 3.2 that the additional quencher has a larger effect on the LER at 0.23 and 0.46 MTR ratio but this becomes negligible at 0.92 MTR ratio. The resist matrix is limiting the action of the external quencher, not necessarily through active chemical interaction but by competitive self-quenching of the material.

The best LER value is obtained at 0.46 MTR ratio and 5\% additional quencher but this is very similar to 0.23 MTR ratio with the same loading of quencher. 
The MTR level variation experiment was also performed for pillar patterning. Pillars were patterned at relaxed pitches between $50 \mathrm{~nm}$ and $100 \mathrm{~nm}$. Results are shown in Fig. 3.3 below.

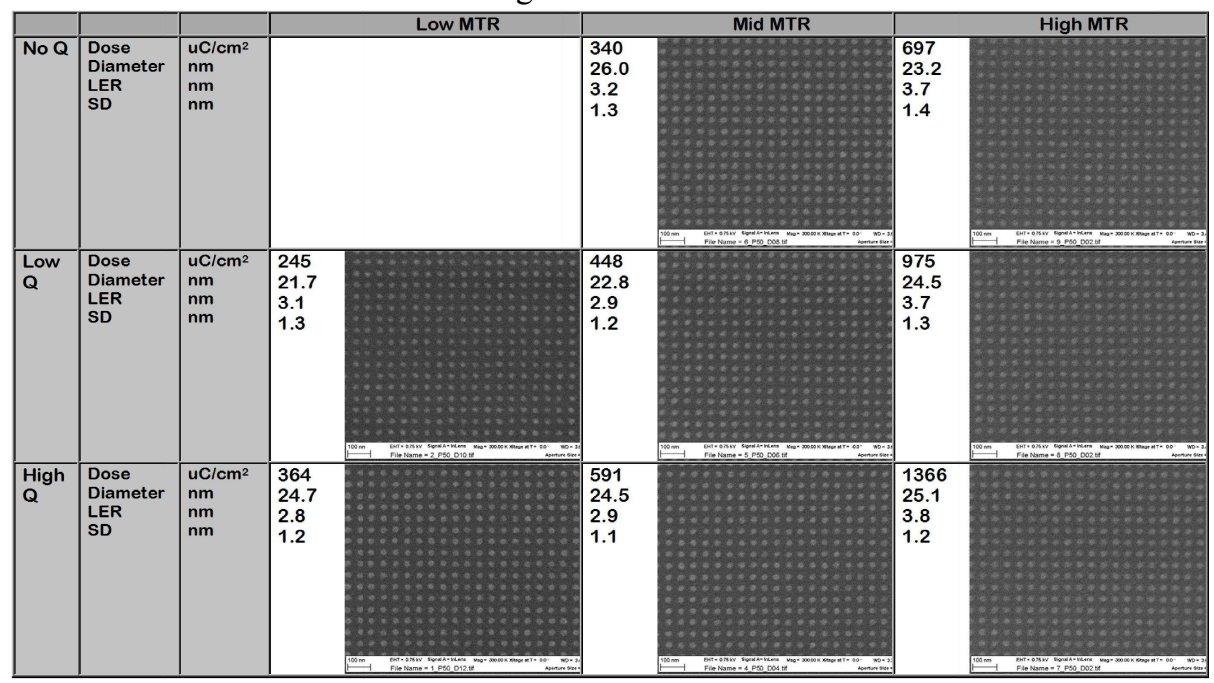

Figure 3.3 $50 \mathrm{~nm}$ pitch pillars on varied MTR level and additional quencher concentration

Figure 3.3 shows a series of pillars with diameters between $22.8 \mathrm{~nm}$ and $26 \mathrm{~nm}$ on a $50 \mathrm{~nm}$ pitch. The different resist samples had various MTR levels and various concentrations of additional quencher, as indicated.

It is observed in Fig. 3.3 that for pillars the increased MTR level doesn't improve the standard deviation from their eccentricity when there is no quencher added. At low concentration of added quencher SD is improved for a medium level of MTR action but SD increases when the MTR action is increased. The trend holds for higher concentration of additional quencher, which shows that an optimum MTR action needs to be used in the system for best uniformity.

Pillars on negative tone resist have more degree of freedom than lines, therefore they are more likely to express defects such as pattern collapse and bridging, which will deform the circular shape and introduce roughness. Therefore we patterned elbow-shaped lines, dense and isolated lines to show the resolution and sensitivity that can be achieved using our resist in electron beam. Figure 3.4 shows dense lines pattern at $28 \mathrm{~nm}$ pitch as well as an isolated line and elbowshaped lines at $28 \mathrm{~nm}$ pitch. The dose required to pattern these features was $303 \mu \mathrm{C} / \mathrm{cm}^{2}$.

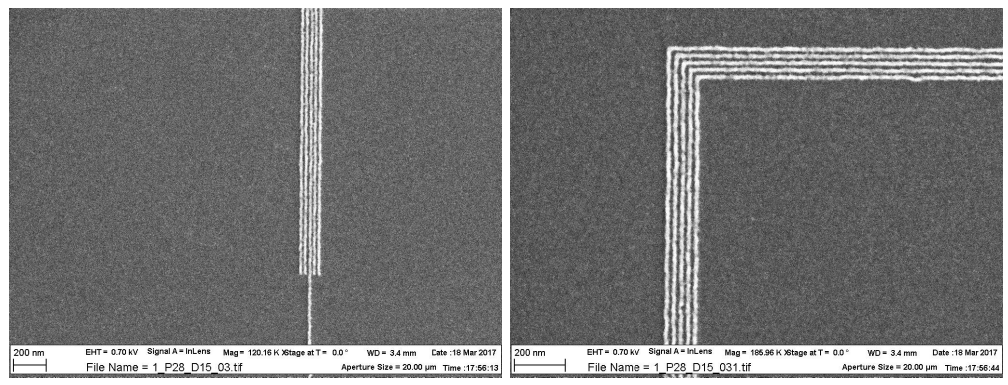

Figure 3.4 Dense lines at $28 \mathrm{~nm}$ pitch, isolated line and elbow-shaped lines on MTR resist, $303 \mu \mathrm{C} / \mathrm{cm}^{2}$

\subsection{MTR for EUV-IL lithography}

Pillars were also patterned with EUV-IL and they are shown in Figure 3.5 below. The dose to mask required to pattern the $18 \mathrm{~nm}$ diameter pillars was $40 \mathrm{~mJ} / \mathrm{cm}^{2} ; 34 \mathrm{~mJ} / \mathrm{cm}^{2}$ was required to pattern $23 \mathrm{~nm}$ diameter pillars; and $24 \mathrm{~mJ} / \mathrm{cm}^{2}$ for 
$24 \mathrm{~nm}$ diameter. The roughness and the non-uniformity of these structures were not calculated, as this would not be an accurate reflection of the material's behaviour but a convolution of the effect of the mask quality and material properties.

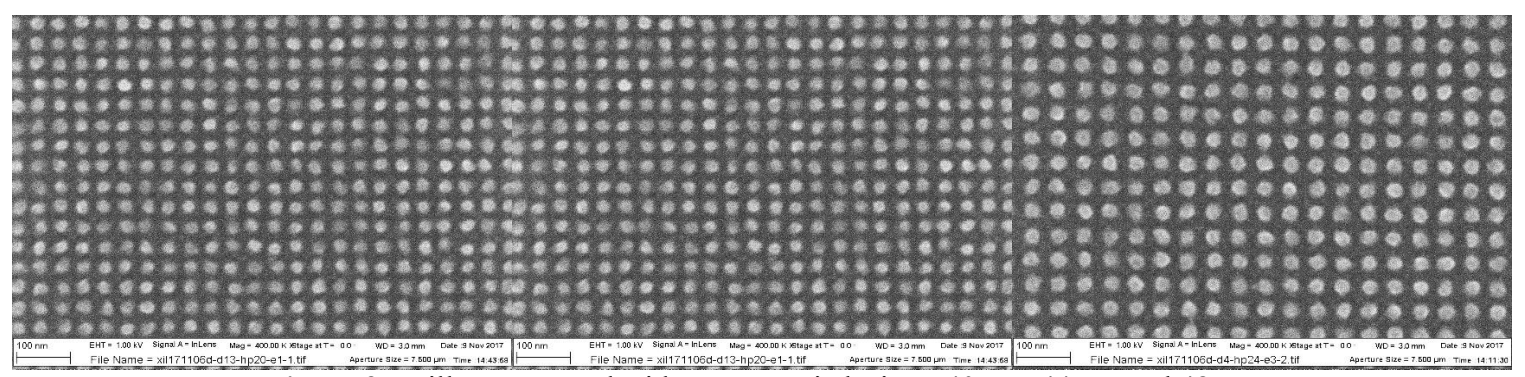

Figure 3.5 Pillars patterned with EUV-IL pitch sizes: $40 \mathrm{~nm}, 44 \mathrm{~nm}$ and $48 \mathrm{~nm}$

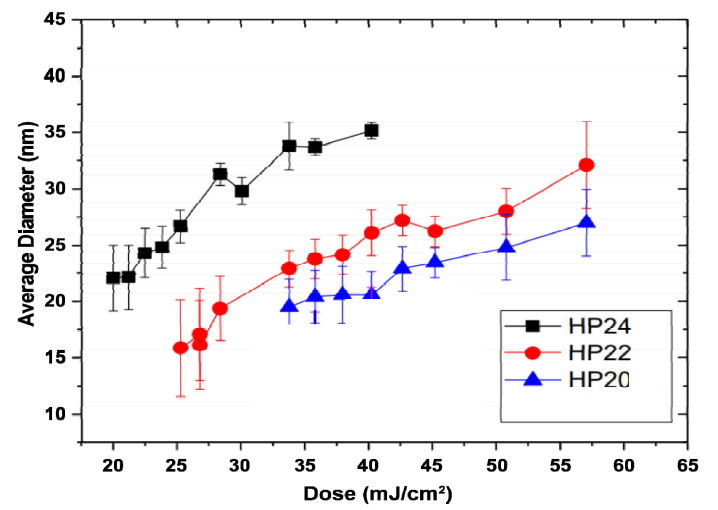

Figure 3.6 Diameter of pillars as a function of the dose to mask

For roughness analysis lines were patterned in EUV at pitch sizes from $44 \mathrm{~nm}$ to $22 \mathrm{~nm}$ and the SEM images analysed using SuMMIT to get the LWR values. Figure 3.7 shows dense lines at $32 \mathrm{~nm}$ pitch size. The left image shows the low MTR action and the right shows the high MTR level. The LWR on the high MTR level lines is reduced from $4.1 \mathrm{~nm}$ to $2.5 \mathrm{~nm}$ at the expense of $15 \%$ in sensitivity.

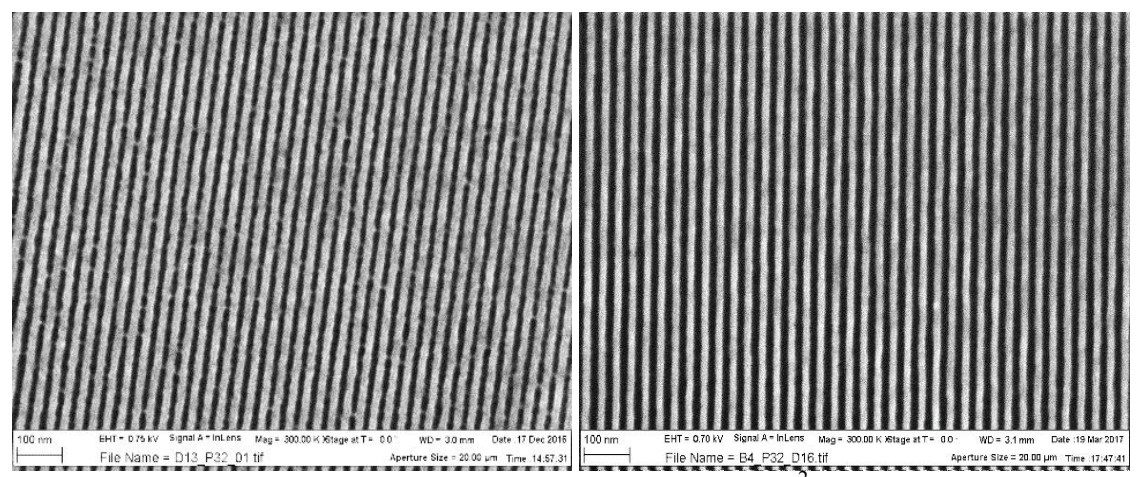

Figure 3.7 Dense lines at $32 \mathrm{~nm}$ pitch in EUV; left-low MTR level, dose $40 \mathrm{~mJ} / \mathrm{cm}^{2}$, LWR $4.1 \mathrm{~nm}$, right- high MTR level, dose 47 $\mathrm{mJ} / \mathrm{cm}^{2}$, LWR $2.5 \mathrm{~nm}$

\section{CONCLUSIONS}

We have shown that the multi-trigger system expresses dose dependent self-quenching behaviour. Varying the MTR component ratio in the system showed that the best LERs are achieved at 0.4 and 0.6 MTR ratios. The resist matrix 
limits the effect of additional quencher in the MTR system and this effect is pronounced at high MTR ratio. We have also shown 21 to $26 \mathrm{~nm}$ diameter pillars patterned with $100 \mathrm{kV}$ electron beam, dense lines on $28 \mathrm{~nm}$ pitch and isolated lines. The lines patterned in EUV showed an improvement of $2.4 \mathrm{~nm}$ in the LWR for high level MTR compared to low level MTR.

\section{ACKNOWLEDGEMENTS}

The authors would like to thank Ms Michaela Vockenhuber from PSI for her assistance with the EUV exposures. Part of this work was performed at Swiss Light Source (SLS), Paul Scherrer Institute, 5232 Villigen, Switzerland. The authors would like to thank the Engineering and Physical Sciences Research Council (EPSRC) for support of this project. The authors thank Irresistible Materials Ltd. and Nano-C for support and provision of resist materials. The Disco DAD 321 wafer dicer used in this research was obtained through the Birmingham Science City provided: Creating and Characterizing Next Generation Advanced Materials, with support from Advantage West Midlands (AWM) and part funded by the European Regional Development Fund (ERDF). C.P. thanks The University of Birmingham for support. This project has received funding from the EU-H2020 research and innovation program under grant agreement No 654360 having benefitted from the access provided by Paul Scherrer Institute in Villigen, Switzerland within the framework of the NFFA-Europe Transnational Access Activity.

\section{REFERENCES}

[1] Popescu C., Frommhold A., McClelland A., Roth J., Ekinci Y., Robinson A.P.G., Proc. SPIE, 10143, 10143V (2017).

[2] Singh V., Satyanarayana V.S.V., Sharma S.K., Ghosh S., Gonsalves K.E., J. Mater. Chem. C, 2, 2118 (2014).

[3] Popescu C., Kazazis D., McClelland A., Dawson G., Roth J., Theis W., Ekinci Y., Robinson A.P.G., "Highresolution EUV lithography using a multi-trigger resist," Proc. SPIE 10583, 105831L (2018).

[4] Montgomery, W., McClelland A., Ure D., Roth J., Robinson A.P.G., "Irresistible Materials multi-trigger resist: the journey towards high volume readiness," Proc SPIE 10143, 1014328 (2017).

[5] Popescu C., McClelland A., Dawson G., Roth J., Kazazis D., Ekinci Y., Theis W., Robinson A.P.G., "Multi-trigger resist for electron beam lithography," Proc SPIE 10446, 1044608 (2017).

[6] Päivänranta B., Langner A., Kirk E., David C., Ekinci Y., "Sub-10 nm patterning using EUV interference lithography," Nanotechnol. 22, 375302 (2011).

[7] Ekinci Y., Vockenhuber M., Terhalle B., Hojeij M., Wang L., Younkin T.R., "Evaluation of resist performance with EUV interference lithography for sub-22 nm patterning," Proc. SPIE 8322, 83220W (2012).

[8] Lorusso G.F., Sutani T., Rutigliani V., van Roey F., Moussa A., Mack, C.A., Naulleau P., Constantoudis V., Ikota M., Ishimoto T., Koshihara S. "The need for line-edge roughness metrology standardization: the imec protocol," Proc. SPIE 10585, 1058512 (2018).

[9] Vesters Y., McClelland A., Popescu C., Dawson G., Roth J., Theis W., de Simone D., Vandenberghe G., Robinson A.P.G, "Multi-trigger resist patterning with ASML NXE3300 EUV scanner," Proc. SPIE 10586, (2018). 